\title{
Design of monitoring system for radioactivity and radiation dose in nuclear medicine
}

\author{
Bin Yang ${ }^{1}$, Xiaowen Zhao ${ }^{1, *}$, Xinwei Wang ${ }^{2}$, Hongtao Zhao ${ }^{1}$, Haixia Yan ${ }^{1}$, Yan $\mathrm{li}^{1}$ and Gang $\mathrm{li}^{1}$ \\ ${ }^{1}$ Technical Physics Institute of Heilongiiang Academy of Sciences, Haerbin, Heilongiiang, 150086, China \\ ${ }^{2}$ Harbin Institute of Technology, Laboratory for Space Environment and Physical Sciences , Haerbin, Heilongjiang, 150006, China
}

\begin{abstract}
In this paper, an intelligent radioactivity and radiation dose monitoring system is designed, which can be used in the field of nuclear medicine. The system can dynamically monitor the body surface radioactivity and radiation dose rate of patients receiving ${ }^{131}$ I radioactive drug treatment, and can identify the personnel who exceed the radiation threshold and send out sound and light alarm. Compared with other monitoring equipment, this system has higher accuracy and can guide the clinical treatment of nuclear medicine scientifically.
\end{abstract}

\section{Introduction}

the national regulations, when the measured activity With the continuous development of nuclear technology, nuclear medicine assisted treatment of tumor technology has also been widely applied. ${ }^{131} \mathrm{I}$ is a kind of medical $\beta$ decay nuclide, its maximum energy is $606 \mathrm{kev}$, half-life is 8.02 days. It can be absorbed and accumulated by thyroid gland, and has good effect in clinical medicine. According to reaches $400 \mathrm{MBq}$ or the radiation dose at 1 $\mathrm{m}$ reaches the public limit of $2.5 \mu \mathrm{Sv} / \mathrm{h}$, the patient can be released from isolation.[1-3] In the process of specific treatment practice, the effective half-life is generally used to estimate the decay and metabolism of ${ }^{131} \mathrm{I}$ in patients, so as to determine the specific discharge time of patients[4-7]. However, this calculation method does not consider the inconsistent metabolism and absorption of radioactive drugs in different patients, which may lead to a large residual radiation dose in patients after the end of the observation period; and in the actual medication process, doctors often increase a certain amount of drugs on top of the basic dosage to achieve better therapeutic effect[8-9]. According to statistics, after the patients were discharged from hospital after treatment, there were still some patients whose body surface radiation dose exceeded the national limit, and there were cases detected by the radioactive security inspection equipment in public places. The highest measured radioactivity was nearly four times higher than the calculated value. Therefore, it is necessary to develop a monitoring system for radioactivity and radiation dose in nuclear medicine.

\section{System design}

\subsection{Structure of the system}

As shown in Figure 1, the architecture of the monitoring system for radioactivity and radiation dose in nuclear medicine is shown in figure 1, which is mainly composed of detection unit and supporting circuit, control and storage core and peripheral modules.

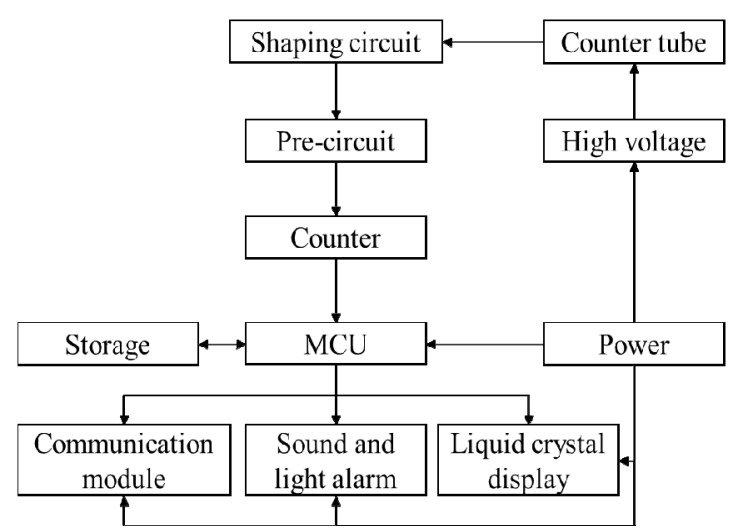

Fig. 1. Structure of the system.

The nuclear detection unit and supporting circuit include GM counter, high voltage power supply module, counter, shaping circuit and front circuit. The control and storage core includes PIC32 single chip microcomputer, internal memory and auxiliary memory. The peripheral module is composed of communication module, sound and light alarm, liquid crystal display and power supply.

The nuclear detection unit and support circuit are mainly used to measure the personnel radiation dose and activity. The control and storage core is mainly responsible for signal processing, data acquisition, storage and processing. The communication module is responsible for the interaction of data and instructions between the upper computer. The sound and light alarm can send out sound and light alarm at the scene when the

\footnotetext{
* Corresponding author: zhaoxiaowen $@$ hljas.cn
} 
radioactivity or radiation dose exceeds the set threshold. The LCD supports three data display modes: radioactive activity, counting rate and radiation dose rate, and can dynamically switch among the three. The power supply is the internal core and external of the system part of the module provides power supply.

\subsection{Functional design}

This system adopts the integrated structure design, which can be directly fixed on the wall through the power plug to achieve the purpose of plug and play. It also integrates an audio-visual alarm and a high-definition LED display screen, which can monitor the radiation dose rate and activity in the monitoring range in real time, conduct onsite sampling and remote data transmission, and reduce the number of medical staff The number of contact between the staff and the treatment patients, reduce the risk of medical staff exposure to radiation, realize remote reading and recording the body dose level of patients after medication, so as to better implement the treatment. The detailed functions of the system are described as follows:

- The radiation dose and activity on the body surface were measured at 1 meter.

- Sound and light alarm function for radiation dose overrun.

- With the function of switching and displaying radiation dose rate, counting rate and radioactivity.

- With the function of parameter storage and reading.

- Support wireless network data transmission function.

- Support remote access control of mobile terminals such as tablet, mobile app, etc.

- Remote setting of alarm threshold and other parameters and automatic timing function.

- Automatic operation and self diagnosis of fault.

\subsection{Working principle}

The high precision GM counter is used as the core part of the nuclear detector of the radioactivity and radiation dose monitoring system of nuclear medicine, and the high voltage required by the GM counter is provided by the high voltage power supply module. When the highspeed gamma photons enter the GM counter, the energy of the gamma photons makes the gas in the GM counter ionize and conduct electricity, which produces a rapid gas discharge phenomenon between the wire electrode and the tube wall, thus outputting a pulse current signal. However, the pulse signal is not conducive to the analysis and processing of the electrical signal, so it is necessary to use the shaping circuit to transform the signal and generate the signal which can be analysed and identified. There is a linear relationship between the counting rate and the radioactive dose rate, and the conversion relationship and the corresponding parameters can be determined by linear regression method; and the radiation dose rate and radioactivity activity can also be converted through a series of changing.

In order to determine the conversion relationship between the counting rate and the radioactive dose rate, the standard source with known activity is generally used to calibrate the radiation monitoring instrument. Considering the influence of statistical fluctuation and error, and eliminating the possibility of negative value in the calculation value of linear equation through the origin, the method of subsection calibration is generally adopted in practical application, which will be introduced in detail later in this paper.

\subsection{Software Design}

The main functions of the system control software are as follows:

- Read configuration, clock initialization, port and module settings.

- The function of signal counting is realized. The counting is converted into dose equivalent and dose rate and processed, including cumulative dose accumulation, data storage and whether sound alarm is given.

- Send and receive data, complete the communication task.

- Control LED display count rate, radiation dose rate, radioactive activity value and alarm threshold.

The overall process is shown in figure 2:

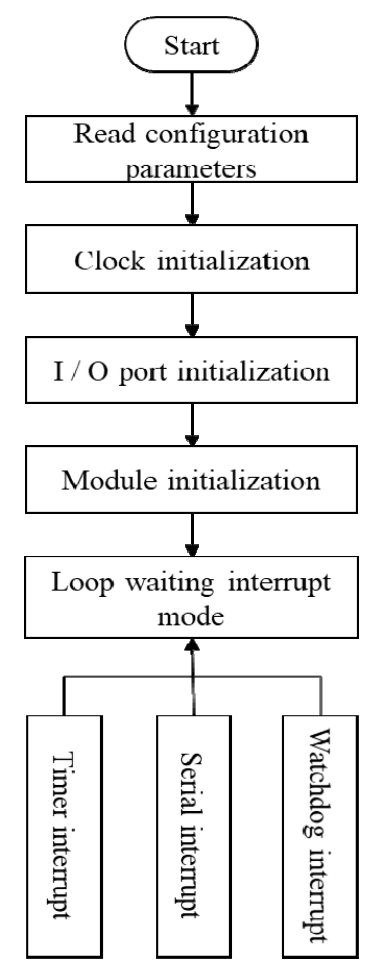

Fig. 2. Program flow chart. 


\subsection{Conversion between radioactivity and radiation dose}

Due to the strong absorption of ${ }^{131} \mathrm{I}$ in human thyroid gland, ${ }^{131} \mathrm{I}$ generally accumulates around the thyroid gland and kills cancer cells through $\beta$ ray ionization. In a certain distance range, the patients treated can be regarded as point source, and the calculation formula of the radiation rate of the point source can be used:

$$
\dot{X}=\frac{A \Gamma}{R^{2}}
$$

In the formula above, $\dot{X}$ is the radiation rate, $A$ is the radioactivity, $\Gamma$ is the gamma radiation rate constant, if the radiation rate is known, it can be deduced from the patient's $A$.

The relationship between absorbed dose rate $\dot{D}$ and radiation rate $\dot{X}$ is as follows:

$$
\dot{D}=8.69 \times 10^{-3} \dot{X}
$$

The relationship between absorbed dose rate $\dot{D}$ and dose equivalent rate $\dot{H}$ is as follows:

$$
\dot{H}=\dot{D} Q N
$$

$N$ is the product of all other correction factors, ICRP specifies $N=1, Q$ is the quality factor, which is generally taken as $Q=1$ in external illumination, so the formula 3 can be further simplified as

$$
\dot{H}=\dot{D}
$$

In general, the dose equivalent rate of patients can be obtained directly by measurement, but the radioactivity is not easy to measure. Therefore, the activity a can be obtained by inverse derivation of formula 1-4

$$
A=\dot{X} \times \frac{R^{2}}{\Gamma}=\frac{\dot{D}}{8.69 \times 10^{-3}} \times \frac{R^{2}}{\Gamma}=\frac{\dot{H}}{8.69 \times 10^{-3}} \times \frac{R^{2}}{\Gamma} \approx \frac{115.1 \dot{H} R^{2}}{\Gamma}
$$

If suppose that the distance $(R=1 \mathrm{~m})$ between the patient and the detector, $\Gamma=2.56 \times 10^{18} \mathrm{C} \cdot \mathrm{m}^{2} / \mathrm{kg}$, then:

$$
A \approx \frac{115.1 \dot{H} R^{2}}{\Gamma}=\frac{115.1 \dot{H} \times(1)^{2}}{2.56 \times 10^{-18}}=4.5 \times 10^{19} \dot{H}
$$

\subsection{Segment calibration}

The pulse count can be converted into the actual radiation dose rate by calibration. Generally, there is a linear relationship between the two values:

$$
f(x)=k x+b
$$

In the above formula, $x$ is the pulse count, $f(x)$ is the radiation dose rate value, $k$ is the slope, and $b$ is the correction parameter.

However, in the practical application, this linear relationship is not suitable for the whole range, and there are often too large deviation between the calculated value and the actual value of a certain section or the negative value of the origin. The method of dividing the measuring range into several intervals and using different $k$ and $b$ to calibrate the corresponding relationship between $x$ and $f(x)$ can better realize the fitting between the calculated value and the true value, and with the increase of the number of segments, the calculation error can be guaranteed within the allowable range.
There are many algorithms for segment calibration. Generally, interpolation method is used to find several points with large linear deviation in the whole range, and then calculate the slope respectively.

Finally, we choose the five segment segmentation method as shown in formula 8

$$
f(x)=\left\{\begin{array}{l}
0.5 x+0.05(0 \leq x<0.1) \\
0.05 x+0.03(0.1 \leq x<15) \\
0.061 x-0.19(15 \leq x<50) \\
0.071 x-0.75(50 \leq x<150) \\
0.081 x-2.23(x \geq 150)
\end{array}\right.
$$

The figure 3 shows that the radiation dose rate calculated by formula 8 falls within the acceptable range parallel to both sides, and in the actual application process, this calibration method can objectively reflect the corresponding relationship between pulse count and radiation dose rate in the whole range.

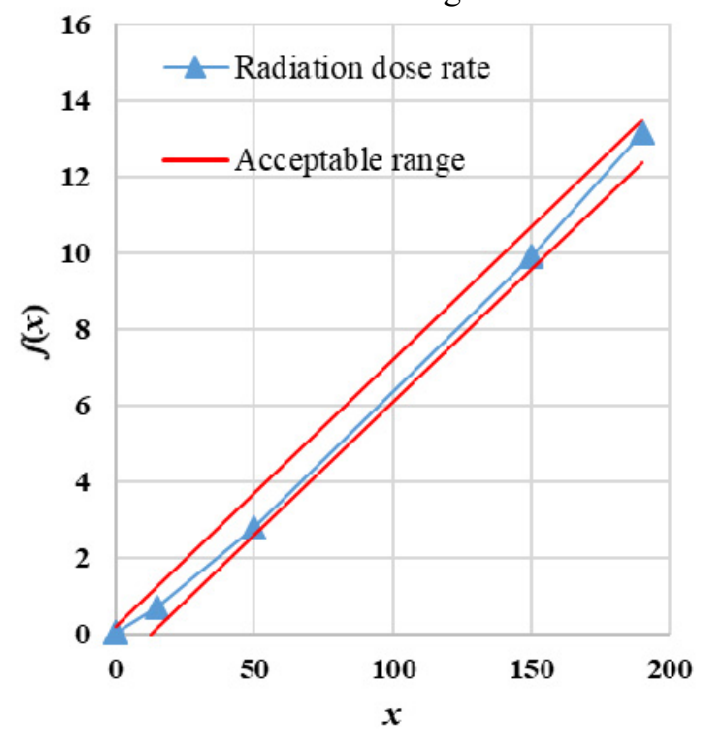

Fig. 3. Results after calibration.

\subsection{Rolling average filtering}

In order to suppress the appearance of the variable value effectively and achieve the purpose of smoothing the data. This paper uses the rolling average filtering method to process the count rate data. The specific methods are as follows: open a data buffer, mainly using the first in first out queue mode, and store 10 sampling data in sequence. Each time a new data is collected, the earliest collected data will be discarded, and then the weighted average value including the new data will be calculated as the current count rate.

$$
N_{\text {count }}=\frac{\left(w_{1} x_{1}+w_{2} x_{2}+\cdots+w_{10} x_{10}\right)}{10}
$$

In the above formula, $N_{\text {count }}$ is the counting rate obtained after weighted average, $x_{i}(1 \leq i \leq 10)$ is the counting rate, and $w_{i}(1 \leq i \leq 10)$ is the weight corresponding to $x_{i}$. The principle of weight setting is: the weight of the newly collected data is higher than that of the data stored in the buffer previously. At the same 
time, the weight is dynamically adjusted according to the frequency of the extreme value. If only one extremely high or very low value appears, the weight is set to the lowest. If there are more than three higher or lower values in succession, the corresponding data weight value will be increased to the highest.

\section{Research \& Results}

In order to verify the reliability and accuracy of the system, the system was compared with the measured values of spherical ionization chamber, 451P, $\gamma$ dose rate monitor and personal dosimeter.

Table 1 shows the measured values of each instrument at $0.05 \mathrm{~m}, 0.5 \mathrm{~m}, 1 \mathrm{~m}$ and $1.5 \mathrm{~m}$ with the standard source placed in the lead tank. Through comparison, it is found that the measurement data of the system within $1 \mathrm{~m}$ are basically consistent with those of spherical ionization chamber, $451 \mathrm{P}$ and other instruments with relatively high measurement accuracy.

Table 1. Comparison of measured values of standard source placed in lead tank.

\begin{tabular}{cccccc}
\hline & \multicolumn{5}{c}{ Different instruments $(\mu \mathrm{Sv} / \mathrm{h})$} \\
$\begin{array}{c}\text { Distance } \\
(\mathrm{m})\end{array}$ & system & $\begin{array}{c}\text { Ionization } \\
\text { chamber }\end{array}$ & $451 \mathrm{P}$ & $\begin{array}{c}\gamma \text { dose rate Personal } \\
\text { monitor }\end{array}$ & dosimeter \\
\hline 0.05 & 3.96 & 3.87 & 3.90 & 4.14 & 4.87 \\
0.5 & 0.43 & 0.38 & 0.41 & 0.52 & 0.60 \\
1 & 0.31 & 0.29 & 0.26 & 0.21 & 0.18 \\
1.5 & 0.22 & 0.15 & 0.16 & 0.14 & 0.10 \\
\hline
\end{tabular}

Table 2 shows the measured values of each instrument at $0.5 \mathrm{~m}, 1 \mathrm{~m}$ and $1.5 \mathrm{~m}$ when the standard source is placed in an open environment. The system also maintains good accuracy.

Table 2. Comparison of measured values of standard source in open environment.

\begin{tabular}{|c|c|c|c|c|c|}
\hline \multirow{2}{*}{$\begin{array}{l}\text { Distance } \\
\text { (m) }\end{array}$} & \multicolumn{5}{|c|}{ Different instruments $(\mu \mathrm{Sv} / \mathrm{h})$} \\
\hline & system & $\begin{array}{l}\text { Ionization } \\
\text { chamber }\end{array}$ & $451 \mathrm{P}$ & $\begin{array}{c}\gamma \text { dose rate } \\
\text { monitor }\end{array}$ & $\begin{array}{l}\text { Personal } \\
\text { dosimeter }\end{array}$ \\
\hline 0.05 & 3.96 & 3.87 & 3.90 & 4.14 & 4.87 \\
\hline 0.5 & 0.43 & 0.38 & 0.41 & 0.52 & 0.60 \\
\hline 1 & 0.31 & 0.29 & 0.26 & 0.21 & 0.18 \\
\hline 1.5 & 0.22 & 0.15 & 0.16 & 0.14 & 0.10 \\
\hline
\end{tabular}

\section{Conclusion}

The monitoring system of radioactivity and radiation dose in nuclear medicine designed in this paper can monitor the body surface radioactivity and radiation dose rate of patients treated with ${ }^{131} \mathrm{I}$ remotely. When the body surface dose or activity exceeds the standard, it can send out sound and light alarm. Compared with other monitoring equipment, the system has higher accuracy, which is of great significance for guiding the clinical treatment of nuclear medicine, reducing unnecessary radiation injury of medical staff and ensuring the health and safety of patients' families and the public.

\section{Acknowledgments}

This paper is one of the phased achievements of the project "research and application of ${ }^{131} \mathrm{I}$ radiation dose distribution of medical nuclide" (YZ2019WL01) funded by the president of Heilongjiang Academy of Sciences.

\section{References}

1. Salvatori M., Luster M. (2010) Radioiodine therapy dosimetry in benign thyroid disease and differentiated thyroid carcinoma. Euro J Nucl Med Mol Imaging, 37(4):821-828.

2. Kobe C., Eschner W.,Wild M. (2010) Radioiodine therapy of benign thyroid disorders: what are the effective thyroidal halflife and uptake of 131I. Nucl Med Commun,31(3): 201-205.

3. Friedman M., Marvin I., Ghesani M. (2002) Interactive software automates personalized radiation safety plans for $\mathrm{Na}^{131} \mathrm{I}$ therapy. Health Phys., 83(4):71-84.

4. Coburn, M., Teates, D., \& Wanebo, H. J. (1994). Recurrent thyroid cancer. Role of surgery versus radioactive iodine $\left({ }^{131} \mathrm{I}\right)$. Annals of surgery, 219(6), 587.

5. Daniel, F, Lima, L., Grando, L., Castro R.., Cordeiro, E., \& Santos C. (2018). Salivary evaluation in radioactive 1131 treated patients with thyroid carcinoma. Acta Odontologica Scandinavica, 76(2), 148-152.

6. Ish-Shalom, S., Durleshter, L., Segal, E., \& Nagler, R. (2008). Sialochemical and oxidative analyses in radioactive I131-treated patients with thyroid carcinoma. European journal of endocrinology, 158(5), 677-682.

7. Franklyn, J., Sheppard M., \& Maisonneuve P. (2005). Thyroid function and mortality in patients treated for hyperthyroidism. Jama, 294(1), 71-80.

8. Alhaj A., Lagarde C., Lobriguito A. (2007) Patient parameters and other radiation safety issues in therapy for thyroid cancer trement. Health Phys., 93:656-666.

9. Feeney D., Jessen, C., Weichselbaum R., Cronk D., \& Anderson K. (2003). Relationship between orally administered dose, surface emission rate for gamma radiation, and urine radioactivity in radioiodinetreated hyperthyroid cats. American journal of veterinary research, 64(10), 1242-1247. 